\title{
UCRL-JRNL-207515
}

LAW RENCE LIVERMORE N A T IO N A L LABORATORY

\section{ADVANCING THE ION BEAM THIN FILM PLANARIZATION PROCESS FOR THE SMOOTHING OF SUBSTRATE PARTICLES}

P. B. Mirkarimi, E. Spiller, S. L. Baker, J. C. Robinson, D. G. Stearns, J. A. Liddle, F. Salmassi, T. Liang, A. R. Stivers

October 27, 2004

Microelectronic Engineering 
This document was prepared as an account of work sponsored by an agency of the United States Government. Neither the United States Government nor the University of California nor any of their employees, makes any warranty, express or implied, or assumes any legal liability or responsibility for the accuracy, completeness, or usefulness of any information, apparatus, product, or process disclosed, or represents that its use would not infringe privately owned rights. Reference herein to any specific commercial product, process, or service by trade name, trademark, manufacturer, or otherwise, does not necessarily constitute or imply its endorsement, recommendation, or favoring by the United States Government or the University of California. The views and opinions of authors expressed herein do not necessarily state or reflect those of the United States Government or the University of California, and shall not be used for advertising or product endorsement purposes. 


\section{Paper entitled}

"Advancing the Ion Beam Thin Film Planarization Process for the Smoothing of Substrate Particles"

Submitted for consideration for publication in the journal

\section{Microelectronic Engineering}

October 2004

UCRL -JRNL-207515 



\title{
Advancing the Ion Beam Thin Film Planarization Process for the Smoothing of Substrate Particles
}

\author{
P.B. Mirkarimi, E. Spiller, S.L. Baker, and J.C. Robinson, \\ Lawrence Livermore National Laboratory, \\ Livermore, CA 94550 \\ D.G. Stearns \\ OS Associates \\ Mountain View, CA. 94040 \\ J.A. Liddle and F. Salmassi \\ Lawrence Berkeley National Laboratory \\ Berkeley, CA. 94720 \\ T. Liang and A.R. Stivers \\ Intel Corporation \\ Santa Clara, CA. 95052
}

\begin{abstract}
For a number of technologies small substrate contaminants are undesirable, and for one technology in particular, extreme ultraviolet lithography (EUVL), they can be a very serious issue. We have demonstrated that the Ion Beam Thin Film Planarization Process, a coating process designed to planarize substrate asperities, can be extended to smooth $\sim 70 \mathrm{~nm}$ and $\sim 80 \mathrm{~nm}$ diameter particles on EUVL reticle substrates to a height of $\sim 0.5 \mathrm{~nm}$, which will render them noncritical in an EUVL printing process. We demonstrate this smoothing process using controlled nanoscale substrate particles and lines fabricated with an e-beam lithography process. The above smoothing process was also modified to yield an excellent reflectance/wavelength uniformity and a good EUV reflectivity for the multilayer, which is required for EUVL reticles. Cross-sectional TEM on a smoothed substrate line defect shows excellent agreement with results obtained from our multilayer growth model.
\end{abstract}




\section{Introduction}

For a number of technologies small substrate contaminants are undesirable, and for one technology in particular, extreme ultraviolet lithography (EUVL), they can be a very serious issue. Extreme ultraviolet lithography ${ }^{1}$ is a leading next-generation lithography technique being developed to continue Moore's law on or after the 2009 timeframe; it is desirable to maintain Moore's law since it has enabled the production of much faster microprocessor chips and higher-capacity memory chips with constant or decreasing costs. Moore's law predicts that the number of transistors or memory bits on integrated circuits will double about every $1.5-2.0$ years $^{2-4}$; this has been achieved primarily by shrinking important features, such as transistor gate lines, to smaller sizes. As the features are reduced in size to several tens of nanometers the wavelength of the light used in the current lithography techniques $(\sim 193 \mathrm{~nm})$ becomes a limiting factor; this makes EUVL, which uses $\sim 13 \mathrm{~nm}$ light, advantageous. One difference between EUVL and previous lithographic technologies is that EUVL requires a reflective reticle; this is due to the fact that substrates with sufficient transmission at extreme ultraviolet wavelengths do not exist. The EUVL reticle blank consists of a substrate coated with a $\mathrm{Mo} / \mathrm{Si}$ multilayer film designed to reflect light with a wavelength of $\sim 13.5 \mathrm{~nm}$ at nearnormal incidence; this multilayer-coated substrate is further processed to produce an absorber pattern on the surface to form the EUVL reticle. In conventional transmission reticles very small particles (tens of $\mathrm{nm}$ in diameter) on the substrate do not result in critical (printable) defects, and hence these substrate particles are not a significant concern. In reflective EUVL reticles very small particles (tens of $\mathrm{nm}$ in diameter) on the substrate that are coated with a Mo/Si multilayer film can nucleate critical phase defects 
in the reflective multilayer coating, which could significantly degrade device performance and reduce the device yield. We have developed a strategy for mitigating the effect of small substrate particles that relies on depositing a Mo/Si multilayer with excellent smoothing properties. Previous work ${ }^{5-7}$ indicates that with a proper selection of the deposition conditions, the smoothing properties of Mo/Si multilayer films can be enhanced with respect to the film's response to substrate asperities. Very recently we demonstrated that the smoothing of substrate particles can be enhanced by using a secondary ion source to etch the Si layers in the deposition of Mo/Si multilayers by ion beam sputtering; we termed this the Ion Beam Thin Film Planarization Process ${ }^{8}$. Using $50 \mathrm{~nm}$ diameter gold nanoparticles randomly deposited on the substrate, we were able to demonstrate a process that smoothed the particles to a height of $\sim 1 \mathrm{~nm}$, which is expected to render them harmless in an EUVL lithography process. Since the completion of this important work ${ }^{9}$ we have made several major advancements which are described in this paper. Firstly, we advanced the process to smooth particles $\sim 70$ and $80 \mathrm{~nm}$ in diameter, which is a significant achievement considering that the particle volume scales as the $\mathrm{D}^{3}$, where $\mathrm{D}$ is the particle diameter ; the $80 \mathrm{~nm}$ particle has over four times the volume of a $50 \mathrm{~nm}$ particle. Secondly, the gold nanoparticle technique ${ }^{10}$, which was the best available technique to date to produce substrate particle defects, required a significant amount of atomic force microscopy time to locate the sparse, randomly oriented particles and the size distribution of the measured particles was a higher than ideal. We have developed and employed a technique to produce controlled arrays of substrate particle defects using a lithographic-based process, saving precious characterization time and also leading to a more tightly controlled distribution of particle sizes. Thirdly, we were able to obtain a 
coating EUV reflectance uniformity of $0.10 \%$ across a $160 \mathrm{~mm}$ diameter substrate, which is particularly impressive considering that there are two potential sources of nonuniformity, the deposition flux profile due to the primary ion source and the etching profile due to the secondary ion source. Lastly, we obtained XTEM images more accurate than any obtained previously; this demonstrates the smoothing process and shows a coneshaped halo in the film above the particle that was predicted by simulations from our improved model of the thin film smoothing process.

\section{Procedure}

\section{a) Fabricating Substrates with NanoParticles}

In order to investigate smoothing in the $\sim 70 \mathrm{~nm}$ particle diameter size regime we first needed to synthesize lithographically-patterned particles with $\sim 70 \mathrm{~nm}$ heights. Such samples, consisting of arrays of nominally tetragonally-shaped particles, were synthesized by electron beam lithography at Lawrence Berkeley National Laboratory (LBNL) on Si substrates. A spin-on-glass resist (HSQ) process was employed and the resist relief features remaining after processing, which consist of highly robust silicon dioxide, serve as the programmed substrate particles.

The programmed substrate particle samples were characterized at Lawrence Livermore National Laboratory and consisted of particles having the same height but a variety of widths. Figure 1 shows an atomic force microscopy (AFM) scan of typical programmed substrate defects. To simplify the discussion we will be referring to 70 high by $70 \mathrm{~nm}$ wide particles as " $70 \mathrm{~nm}$ diameter" particles, where the reader should be aware 
that the particles are not spherical (although there is some rounding of the edges such that the particles have a spherical element to them).

The AFM results are listed in Table 1. For the $70 \mathrm{~nm}$ widths the measured defect height was $71.1 \mathrm{~nm}$ with a standard deviation of $0.85 \mathrm{~nm}$. The $50 \mathrm{~nm}$ and $100 \mathrm{~nm}$ widths yielded similar height numbers, $70.8 \mathrm{~nm}$ and $71.6 \mathrm{~nm}$, respectively. Note that this is a significantly smaller standard deviation in the measured heights than what we reported previously ${ }^{10}$ using the gold nanoparticle technique. These measurements were performed on a Digital Instruments Atomic Force Microscope (AFM). This AFM has shown good agreement with results from another in-house AFM which with a different operator. During the period of time over which the AFM measurements in this report were performed, we tested this AFM against a similar AFM at LBNL using the programmed substrate defects and also against a NIST-certified standard. There was excellent agreement in all cases.

We also synthesized programmed substrate defects with heights closer to $80 \mathrm{~nm}$; these are shown in Table 2.

\section{b.) Multilayer Smoothing}

The Mo/Si multilayer coatings have been deposited using ion beam sputtering in the system shown schematically in Figure 2. The Mo/Si multilayer consists of 50.5 bilayer pairs. A bilayer pair is defined as one Mo layer plus one Si layer, where the bilayer thickness is $\sim 7 \mathrm{~nm}$ and the Mo is $\sim 40 \%$ of the bilayer pair thickness. We found previously that etching of the Si layers during the deposition process significantly enhances the smoothing properties of the coatings and this process, illustrated schematically in Figure 3, is employed in this work. The Mo and Si layers are deposited 
at normal incidence; however, for the Si layers an additional $\sim 1-3 \mathrm{~nm}$ of material is deposited and then etched away, also at near-normal incidence. When excellent uniformity is required the Mo and Si layers are deposited at an off-normal angle, which is discussed later on (near Figure 9 in the paper).

The primary ion source beam energy was $600 \mathrm{eV}$ (i.e., the ion used to generate the deposition flux from the target) and the energy of the secondary ion source (used for etching of the Si layers) was $150-250 \mathrm{eV}$. Ar was used as the source gas for both ion sources.

\section{c) Characterization and Simulations}

After the deposition of the $\mathrm{Mo} / \mathrm{Si}$ multilayer coatings the samples were measured again with AFM to characterize the height and width of the defects at the film surface. The values were determined by averaging over a number of defects, and the quoted error is the standard deviation of the values. Specifics about process parameters for particular coating runs, when and where appropriate, are listed throughout the tables and text.

Cross-sectional transmission electron microscopy (XTEM) samples were made by locating and sectioning the area of interest with a FEI 835 dual beam focused ion beam tool and then imaging the section in a JEOL 2010 TEM. The cross sections are made by first depositing a protective platinum layer using both electron beam and ion beam assisted deposition processes. The cross section is then cut and thinned with the ion beam to a thickness of $\sim 0.2-0.3$ microns. A final cleaning takes place using a low voltage ion beam to remove any material that may have re-deposited during sectioning. The section is placed on a copper grid and imaged in the TEM at a voltage of $200 \mathrm{kV}$. 
The simulations of defect growth have been performed using a nonlinear continuum model presented in detail previously ${ }^{11}$. In this model we describe the multilayer film growth as a sequence of deposition and etch steps applied to a surface that has an initial profile of the substrate defect. The deposition and etch processes are constrained to be near normal incidence so that shadowing effects can be neglected. At each growth step the surface is allowed to relax by applying a linear Fourier filter that preferentially removes the high frequency components. This linear filter accounts for a certain amount of in plane mobility of the adatoms due to, for example, surface diffusion that tends to locally smooth the surface. We also include at each step a nonlinear process. In the deposition step this process is the contraction of the film due to the interdiffusion of Si and Mo at the interface to form a thin layer of intermetallic silicide. In the etch step the nonlinear growth arises from the complex dependence of the etch rate on the local angle of incidence. Both of these nonlinear processes have the effect of preferentially eroding the sides of the defect where the surface slope is largest. This causes bump-like defects to narrow. The simulation generates the two-dimensional profiles of the successive interfaces in the multilayer stack, which can be directly compared to a TEM cross-section of the film.

\section{d) AFM Data Analysis}

Our primary source of experimental measurements of defects is atomic force microscopy (AFM). AFM provides a two-dimensional topographical profile of the surface of the film. The AFM scan is then analyzed using a custom IDL routine called "IMAGEPLUS". This routine provides a variety of useful functions including finding and 
counting defects, determining their height, width and volume, taking profiles, and generating a power spectral density of the surface roughness. As our smoothing process has improved the defect profiles have decreased in height to the point where they are approaching the peak-to-valley noise fluctuations produced by the surface roughness. Consequently we have had to develop a more sophisticated algorithm for analyzing the defect profile, and extracting the height and width information. The nature of the problem can be seen in Fig.4, where we show the profile of a defect nucleated by a 70 x $70 \mathrm{~nm}$ particle and smoothed to less than $1 \mathrm{~nm}$ in height using a $150 \mathrm{~V}$ etch step. The peak-tovalley noise in the raw AFM scan is comparable to the height of the defect. To analyze this data it is necessary to first apply a local averaging algorithm to the AFM scan. Specifically, we convolve the image with a 9-pixel-wide rectangle function. The effect of this averaging on the defect profile is shown as the red line in Fig. 4. It is clear that this procedure is only valid if the width of the defect is much greater than the convolving rectangle. In practice this means that there must be only a few nanometers per pixel; for example, a relatively small $1 \times 1 \mu \mathrm{m}$ AFM scan should be sufficient with its 512 points. This suggests that more AFM characterization time will be required with this new analysis method, since less data is obtained via $1 \times 1 \mu \mathrm{m}$ AFM scans than in $5 \times 5 \mu \mathrm{m}$ AFM scans.

The height of the defect is determined from the averaged profile. To obtain a value for the full-width-at-half-maximum (FWHM) the algorithm finds all of the pixels in the defect that exceed half the maximum height. The total area A of these pixels is used to estimate the width according to the formula FWHM $=1 / 2(\mathrm{~A} / \Pi)^{1 / 2}$. Although the averaging process provides an improved method of obtaining height and width values, it 
must be recognized that these are still subject to error due to the noise produced by the surface roughness. Hence quantitative comparisons of defect measurements when the heights are less than $\sim 1 \mathrm{~nm}$ become more problematic, and the FWHM values are a particular issue.

We currently believe that the most accurate method is to obtain $1 \times 1 \mu \mathrm{m}$ AFM scans and use the newly developed method (described above) for the smoothing analysis. However, we discovered these data analysis issues in compiling this paper and most of our data is in the form of $5 \times 5 \mu \mathrm{m}$ scans. We will present data analyzed by both methods and note this when and where applicable in the paper. The older method, employing all of the data, is labeled as analysis method 1. The newly developed method, which is more accurate but has fewer data points to use, is termed analysis method 2. It should be noted that whether method 1 or 2 is used do not affect the basic conclusions of EUVL printability since the smoothed heights of our $70 \mathrm{~nm}$ and $80 \mathrm{~nm}$ substrate particles are so small that it doesn't matter too much whether we are overestimating the smoothed height by a few tenths of a nm or underestimating the smoothed widths by tens of nm's using the old analysis method. This is due to the shape of the printability curve, shown in Figure 7, which is a very weak function of FWHM when the smoothed heights are sufficiently small.

\section{Results and Discussion}

Using the ion beam thin film planarization process we were able to smooth $70 \mathrm{~nm}$ diameter particles to $\sim 0.6 \mathrm{~nm}$ in height. The results are shown in Figure 5 below. We did two runs, one where the Ar ion energy was $250 \mathrm{eV}$ for the Si etch (V1505C) and one 
with a $150 \mathrm{eV}$ Ar ion energy, for the Si etch. The results, as a function of the initial starting width of the substrate defect, is also shown in Table 3. There is no major difference in the smoothing for the two etch energies although he lower etch energy of $150 \mathrm{eV}$ may result in a minor improvement in smoothing over the larger etch energy of $250 \mathrm{eV}$.

In Figure 5 we also compare the smoothing to what is achieved by a standard Mo/Si coating process (employing a normal incidence deposition flux) with no Si etching (i.e., no enhanced smoothing); in this case the $70 \mathrm{~nm}$ particles are only smoothed to mean height of $39 \mathrm{~nm}$ using analysis method 1.

Since this work is aimed at developing a process which can eventually be implemented in mask blank manufacturing, repeatability is important. One set of conditions, those used for run V1505C, was repeated three additional times over a period of $\sim 6$ months. This is shown in Table 4 and Figure 6 below. Three of the four were virtually identical and one of the runs (V1595) showed less smoothing but the height was still less than $0.9 \mathrm{~nm}$.

The height and FWHM of the smoothed $70 \mathrm{~nm}$ particle defects are such that the defects should be nonprintable in an EUVL stepper. This is shown in Figure 7 where the data for a typical sample, V1505C, is compared to a EUVL printability curve generated from the single surface approximation ${ }^{12}$ for an EUVL stepper with multilayer phase defects in proximity to $25 \mathrm{~nm}$ lines; the simulations assume NA $=0.25$ and a $20 \% \mathrm{CD}$ variation criteria. The smoothed particle heights are so low that they should remain non printable regardless of whether data analysis methods 1 or 2 are used and even if the CD variation is reduced further. 
In addition to particle-like defects our substrate defect pattern contains lines. As with the particle-like defects the height of the lines are all at a fixed value, say $70 \mathrm{~nm}$ for example, and the widths vary from line to line. We characterized the smoothed lines on sample V1607 from Table 4 by TEM. The lines have a particular advantage with respect to the XTEM analysis since (i) lines are easier to locate for XTEM sectioning and (ii) unlike for particles, with lines there is little risk of having disturbed and undisturbed layers appearing at the same location in the TEM image (since it's a two dimensional and not a three dimensional defect). The TEM cross-section is shown in Fig.8a. Note that on this sample there was a very thin $\mathrm{Si} / \mathrm{Mo} / \mathrm{Si}$ buffer layer deposited prior to the multilayer, which produced the "double" Si layer seen at the bottom of the stack; the Mo layer helps enhance the contrast for TEM imaging. We also show for comparison in Fig. 8b a simulation of the defect smoothing obtained from our modeling code, which includes the strong nonlinear effects of the angle-dependent etch rate ${ }^{11}$. The XTEM image dramatically shows how the sides of the line narrow and collapse, producing a hat-shaped morphology as predicted by the simulation. The dark lines that make up the hat-like profile are believed due to (i) Mo and Si becoming very thin and intermixing and (ii) that region becoming Mo-rich due to the Si etching more than the Mo. The simulation also captures the experimental observation that there is discernable layering within the hat, a subtle effect. It is clear that most of the smoothing occurs in the first 10 layers - a result that could not be inferred from the AFM measurement of the top surface. We also note that there is no apparent damage to this sample by the FIB preparation process. Another important observation obtained from Fig $8 \mathrm{a}$ is that there is no significant erosion of the substrate particle observed from our smoothing process. In Fig. 8b our simulation of the 
defect smoothing is of a line having an initial height of $70 \mathrm{~nm}$ and width of $70 \mathrm{~nm}$. The final multilayer structure is composed of 50 bilayers, each consisting of $3.0 \mathrm{~nm}$ of Mo and $4.5 \mathrm{~nm}$ of $\mathrm{Si}$. An additional $\sim 1.5 \mathrm{~nm}$ of $\mathrm{Si}$ is deposited and etched at each bilayer using a normal incidence Ar ion beam operating at $150 \mathrm{~V}$ and $200 \mathrm{~mA}$. The linear relaxation parameters of the deposition step are $v_{2}=0.6 \mathrm{~nm}$ and $v_{4}=2.0 \mathrm{~nm}^{3}$. The corresponding relaxation parameters for the etch step are $v_{1}=0.6$ and $v_{4}=10.0 \mathrm{~nm}^{3}$. The growth model also includes the nonlinear effects of the silicide formation at the interfaces and the dependence of the etch rate on the incidence angle. For this simulation we have assumed that the final thickness of the silicide interlayer is $0.8 \mathrm{~nm}$. The dependence of the local etch rate on the angle of incidence $\theta$ is given by,

$$
\mathrm{R}(\theta)=0.015 \times(\cos \theta)^{1.15} \exp \left[-1.86\left((\cos \theta)^{4.43}-1\right)\right] \quad \text { in } \mathrm{nm} / \mathrm{s}
$$

The simulation was performed in 1+1 dimensions (line defect) over a domain of $400 \mathrm{~nm}$ consisting of 200 points.

The EUV lithography tools are expected to require good illumination uniformity on the resist-coated Si wafer. Part of the illumination uniformity error budget is allotted to the mask, which results in a stringent reflectance uniformity requirement of $< \pm 1 \%$ $3 \sigma^{13}$. It can be advantageous to have a smoothing layer that is simultaneously the reflective layer; for this to occur one must be able to deposit a smoothing layer that (i) meets the thickness uniformity requirements across the reticle and (ii) the smoothing layer must have a reasonably high EUV reflectivity 
After several calibration runs, V1612 was deposited with a process that achieves excellent smoothing and uniformity simultaneously; uniformity results for the run are shown in Figure 9 below. The small reflectivity variation (0.10\%) is well within the specifications for the mask blank. Since the substrate is spun about its center during deposition, the $0.10 \%$ variation across the $80 \mathrm{~mm}$ radius can be translated to a variation of $0.10 \%$ across a $160 \mathrm{~mm}$ diameter (or $0.30 \% 3 \sigma$ across the $160 \mathrm{~mm}$ diameter). This uniformity is comparable to the best uniformity values achieved previously without ion etching $^{13}$; this demonstrates that the improved ability of the ion beam thin film planarization process to smooth larger substrate particles was achieved without a degradation in coating uniformity. The smoothing results using this high-uniformity process is shown in Figure 10. The slight bump turns into a slight depression with smoothing when the depo step is at off-normal incidence; this is consistent with results obtained last year when the $50 \mathrm{~nm}$ particle smoothing process was coupled with a high uniformity process ${ }^{8}$.

After demonstrating that the process works for smoothing $70 \mathrm{~nm}$ particles we checked if it could be easily extended to smooth $80 \mathrm{~nm}$ particles. It should be noted that while the increase in particle radius (r) from $70 \mathrm{~nm}$ to $80 \mathrm{~nm}$ is only $14 \%$, the increase is particle volume $(\mathrm{V})$ is $66 \%\left(\right.$ since $\mathrm{V} \sim \mathrm{r}^{3}$ ), and hence $80 \mathrm{~nm}$ particles should be more challenging to smooth than one might first think. We increased the etch time for the Si layers from our usual $109 \mathrm{sec}$ to $218 \mathrm{sec}$ in run V1616 in order to see if $80 \mathrm{~nm}$ particles could be smoothed below the printability threshold. We were able to smooth $80 \mathrm{~nm}$ (height/width) substrate particles to a mean height of $0.50 \mathrm{~nm}$ and a mean FWHM of 55 $\mathrm{nm}$ for the 8 defects that were characterized using analysis method 1. Using analysis 
method 2 on a single characterized defect we obtained a height of $0.37 \mathrm{~nm}$ and a FWHM of $174 \mathrm{~nm}$. Note that for these very small smoothed particle heights the resulting widths differ significantly depending upon whether data analysis method 1 or 2 is used. Independent of the analysis method the $80 \mathrm{~nm}$ particles were smoothed below the printability threshold curve that was shown in Figure 8 previously. An AFM crosssectional profile of a representative smoothed $80 \mathrm{~nm}$ particle is shown in Figure 11 along with profile of a particle in which a standard multilayer coating (with no enhanced smoothing) was deposited. The smoothing was significantly less when Si etching was not employed; an $\sim 80 \mathrm{~nm}$ particle was smoothed to only $\sim 56 \mathrm{~nm}$ without enhanced smoothing.

We also performed a run, V1620, in which a $164 \mathrm{sec}$ etch was used, to see if 80 $\mathrm{nm}$ particle smoothing could be achieved with an etch time shorter than $218 \mathrm{sec}$ per layer. For V1620 the mean defect height and FWHM for the smoothed $80 \mathrm{~nm}$ diameter particles was $1.0 \mathrm{~nm}$ and $182 \mathrm{~nm}$, respectively, using data analysis method 1 on 8 characterized defects; employing analysis method 2 on a single characterized defect we obtained a height of $0.81 \mathrm{~nm}$ with a FWHM of $196 \mathrm{~nm}$. Note that for this somewhat larger height the difference in FWHM values obtained between data analysis methods 1 and 2 is not large and in general these height/FWHM values still represents a significant level of particle smoothing. Using our estimated etch rates of $0.014 \mathrm{~nm} / \mathrm{sec}$ for V1615, V1616, and V1620, etch times of 109, 218, and $164 \mathrm{sec}$ correspond to 1.5, 3.0, and $2.3 \mathrm{~nm}$ of Si etched per Si layer.

Estimated processing times for V1615, V1616, and V1620 are 5.25, 6.75, and 6.0 hours respectively, which will likely need to be reduced to increase mask blank tool 
throughput and reduce mask blank costs. We are optimistic that these deposition times could be reduced with further effort. For example, using higher etch energies, using larger mass inert gas ions for etching, etc, could potentially reduce the etch-related component of the process time. If the process time can be reduced significantly then it may be possible to extend the technique to smooth $90 \mathrm{~nm}$ and $100 \mathrm{~nm}$ diameter particles

\section{Conclusions}

We have demonstrated that the Ion Beam Thin Film Planarization Process, a coating process designed to planarize substrate asperities, can be extended to smooth $\sim 70$ $\mathrm{nm}$ and $\sim 80 \mathrm{~nm}$ diameter particles on EUVL reticle substrates to a height of $\sim 0.5 \mathrm{~nm}$, which will render them noncritical in an EUVL printing process according to EUVL printability modeling. We demonstrate this smoothing process using controlled nanoscale substrate particles and lines fabricated with an e-beam lithography process, which we find preferable to the gold nanoparticle process used and reported previosly. The above smoothing process was also modified to yield excellent reflectance/wavelength uniformity $(0.10 \% 1 \sigma$ variation in EUV reflectivity over a $160 \mathrm{~mm}$ diameter) and good EUV reflectivity (66.2\%), which are also required for EUVL reticles. Cross-sectional TEM on a smoothed substrate line defect shows excellent agreement with results obtained from our multilayer growth model. Coating process times to smooth the $80 \mathrm{~nm}$ substrate particles are up to $\sim 6$ hours; this will need to be reduced however there is a pathway to reduce this time. 


\section{Acknowledgements}

This work was performed under the auspices of the U.S. Department of Energy by the University of California Lawrence Livermore National Laboratory under contract W7405-Eng-48. Funding was provided by Intel Corporation through a work-for-others agreement.

\section{References}

1. P. B. Mirkarimi, "Extreme Ultraviolet Nanolithography," in Encyclopedia of Nanoscience and Nanotechnology, H. S. Nalwa, ed. (American Scientific, 2004), pp. 297-306.

2. G. E. Moore, "Cramming more components onto integrated circuits," Electronics 38(8), (1965).

3. G. Stix, "Getting More from Moore's," Scientific American, April 2001.

4. P. Gargini, "Worldwide technologies and the ITRS in the current economic climate," Proceedings of SPIE 4688, 25-28 (2002).

5. P. B. Mirkarimi and D. G. Stearns, "Investigating the growth of localized defects in thin films using gold nanospheres," Appl. Phys. Lett. 77, 2243-2245 (2000).

6. P. B. Mirkarimi, S. L. Baker, M. A. Wall, P. A. Kearney, and D. G. Stearns, "Defects from substrate particles depend on the sputter deposition process," Solid State Technol. 43(11), 95-99 (2000).

7. P. B. Mirkarimi, E. A. Spiller, D. G. Stearns, V. Sperry, and S. L. Baker, "An IonAssisted Mo/Si Deposition Process for Planarizing Reticle Substrates for Extreme Ultraviolet Lithography," IEEE J. Quant. Elec. 37, 1514-1516 (2001).

8. P. B. Mirkarimi, E. Spiller, S. L. Baker, V. Sperry, D. G. Stearns, and E. M. Gullikson, "Developing a viable multilayer coating process for extreme ultraviolet lithography reticles," J. Microlith., Microfab., Microsyst. 3, 140-145 (2004).

9. R\&D Magazine, 45 (9), 66-66 (2003).

10. P. B. Mirkarimi, S. L. Baker, and D. G. Stearns, "A technique employing gold nanospheres to study defect evolution in thin films," J. Vac. Sci. Technol. B 19, 628-633 (2001).

11. D. G. Stearns, P. B. Mirkarimi, and E. Spiller, "Localized defects in multilayer coatings," Thin Solid Films 446, 37-49 (2004).

12. E. M. Gullikson, C. Cejan, D. G. Stearns, P. B. Mirkarimi, and D. W. Sweeney, "A Practical Approach for Modeling EUVL Mask Defects," J. Vac. Sci. Technol. B 20, 81-86 (2001).

13. S. D. Hector, E. M. Gullikson, P. Mirkarimi, E. Spiller, P. Kearney, and J. Folta, "Multilayer coating requirements for extreme ultraviolet masks," Proceedings of SPIE 4562(2001). 


\section{Tables}

Table 1. Results for the programmed substrate defect sample that was targeted to have $\sim 70 \mathrm{~nm}$ high defects.

\begin{tabular}{|c|l|l|l|}
\hline $\begin{array}{l}\text { Sample } \\
\text { (uncoated) }\end{array}$ & $\begin{array}{l}\text { Width } \\
(\mathrm{nm})\end{array}$ & $\begin{array}{l}\text { Mean } \\
\text { height } \\
(\mathrm{nm})\end{array}$ & $\begin{array}{l}\text { Number of } \\
\text { data points }\end{array}$ \\
\hline PD 1467 & $\sim 50$ & $70.8 \pm 1.0$ & 17 \\
\hline$"$ & $\sim 70$ & $71.1 \pm 0.85$ & 15 \\
\hline$"$ & $\sim 100$ & $71.6 \pm 1.2$ & 9 \\
\hline
\end{tabular}

Table 2. Results for the programmed substrate defect sample that was targeted to have $\sim 80 \mathrm{~nm}$ high defects.

\begin{tabular}{|c|l|l|l|}
\hline $\begin{array}{l}\text { Sample } \\
\text { (uncoated) }\end{array}$ & $\begin{array}{l}\text { Width } \\
(\mathrm{nm})\end{array}$ & $\begin{array}{l}\text { Mean } \\
\text { height } \\
(\mathrm{nm})\end{array}$ & $\begin{array}{l}\text { Number of } \\
\text { data points }\end{array}$ \\
\hline PD 2039 & $\sim 70$ & $79.2 \pm 0.9$ & 9 \\
\hline$"$ & $\sim 90$ & $79.6 \pm 0.5$ & 9 \\
\hline
\end{tabular}


Table 3. Results for V1504C and V1505C which utilized Ar ion etch energies of $250 \mathrm{eV}$ and $150 \mathrm{eV}$ respectively. Defect substrates PD1467 were used and the approximate height and width of the particles before coating were $71 \mathrm{~nm}$ and 70 $\mathrm{nm}$ respectively

\begin{tabular}{|l|l|l|l|c|c|c|c|}
\hline $\begin{array}{l}\text { Sample } \\
\text { (after } \\
\text { coating) }\end{array}$ & $\begin{array}{l}\text { Etch time } \\
\text { per Si } \\
\text { layer } \\
\text { (sec) }\end{array}$ & $\begin{array}{l}\text { Mean height } \\
\text { after coating } \\
(\mathrm{nm})\end{array}$ & $\begin{array}{l}\text { Mean } \\
\text { FWHM } \\
\text { after } \\
\text { coating } \\
\text { (nm) }\end{array}$ & $\begin{array}{l}\text { Roughness } \\
\text { after } \\
\text { coating } \\
\text { (nm,rms })\end{array}$ & $\begin{array}{l}\text { \# of } \\
\text { data } \\
\text { points }\end{array}$ & $\begin{array}{l}\text { Data } \\
\text { Analysis } \\
\text { Method }\end{array}$ & Comments \\
\hline $\begin{array}{l}\text { V1504 } \\
\text { C }\end{array}$ & 27 & $0.93 \pm 0.08$ & 92 & 0.084 & 5 & 1 & $250 \mathrm{eV}$ etch, \\
\hline $\begin{array}{l}\text { V1504 } \\
\text { C }\end{array}$ & “ & 0.67 & 166 & “ & 1 & 2 & " \\
\hline $\begin{array}{l}\text { V1505 } \\
\text { C }\end{array}$ & 109 & $0.60 \pm 0.10$ & 64 & 0.088 & 5 & 1 & $150 \mathrm{eV}$ etch, \\
\hline $\begin{array}{l}\text { V1505 } \\
\text { C }\end{array}$ & “ & 0.53 & 144 & $*$ & 1 & 2 & " \\
\hline
\end{tabular}

Table 4. Smoothing results for repeated use of the V1505C process. The data was analyzed using data analysis method 1.

\begin{tabular}{|l|l|c|}
\hline Sample \# & $\begin{array}{l}\text { Mean Height } \pm \\
\text { Std.Dev. }\end{array}$ & $\begin{array}{l}\text { \# data } \\
\text { points }\end{array}$ \\
\hline V1505C & $0.60 \pm 0.10 \mathrm{~nm}$ & 5 \\
\hline V1561 & $0.54 \pm 0.10 \mathrm{~nm}$ & 8 \\
\hline V1607 & $0.49 \pm 0.25 \mathrm{~nm}$ & 4 \\
\hline
\end{tabular}




\section{Figure Captions}

Figure 1. Atomic force microscopy image of a programmed defect array prior to smoothing.

Figure 2. Schematic diagram of the Ion Beam sputter Deposition (IBD) tool used to synthesize the Mo/Si multilayer coatings.

Figure 3. Illustration of the ion-assisted procedure used to deposit the Mo/Si multilayer coatings.

Figure 4. Profile of the AFM measurement of a defect nucleated by a $70 \times 70 \mathrm{~nm}$ particle and smoothed using a $150 \mathrm{~V}$ etch step. The AFM data is a $1 \times 1 \mu \mathrm{m}$ image having 512 pixels per scan. The black line is the raw data and the red line corresponds to averaging the image by convolving with a 9-pixel-wide rectangle.

Figure 5. Cross-sectional surface profile as measured by atomic force microscopy for 70 $\mathrm{nm}$ diameter substrate particles (a) before and after smoothing, and (b) a closeup of the surface profile after smoothing.

Figure 6. Repeatibility of smoothing data using V1505-like conditions.

Figure 7. Theoretical curve for the printability of a Mo/Si multilayer defect as a function of the defect height and full-width-at-half maximum. Experimental values for V1505C show that the smoothed particle should be rendered nonprintable regardless of the data analysis method employed. Printability curve was generated for defects in proximity to $25 \mathrm{~nm}$ lines assuming a numerical aperture of 0.25 and a $20 \%$ CD variation criteria [see E.M. Gullikson et al., JVST B 20, 81-86 (2001) for details on the EUVL printability model].

Figure 8. (a) Cross-sectional TEM micrograph of a $70 \mathrm{~nm} \times 70 \mathrm{~nm}$ line that was smoothed in multilayer deposition run V1607 and (b) Simulations of the smoothing of the substrate line using the multilayer growth model.

Figure 9. The (a) normalized thickness and (b) peak EUV reflectivity of Mo/Si films as a 
function of the distance from the center for conditions under which $70 \mathrm{~nm}$ particles were smoothed (see next Figure).

Figure 10. Cross-sectional surface profile as measured by atomic force microscopy for 70 $\mathrm{nm}$ diameter substrate particles (a) before and after smoothing, and (b) a close-up of the surface profile after smoothing. Note that under the deposition conditions for optimal uniformity that instead of the small bump observed in Figure $5 \mathrm{~b}$ we obtain a small depression after smoothing.

Figure 11. Cross-sectional surface profile as measured by atomic force microscopy for 80 $\mathrm{nm}$ diameter substrate particles (a) before and after smoothing, and (b) closeup of the surface profile after smoothing. 
Figures

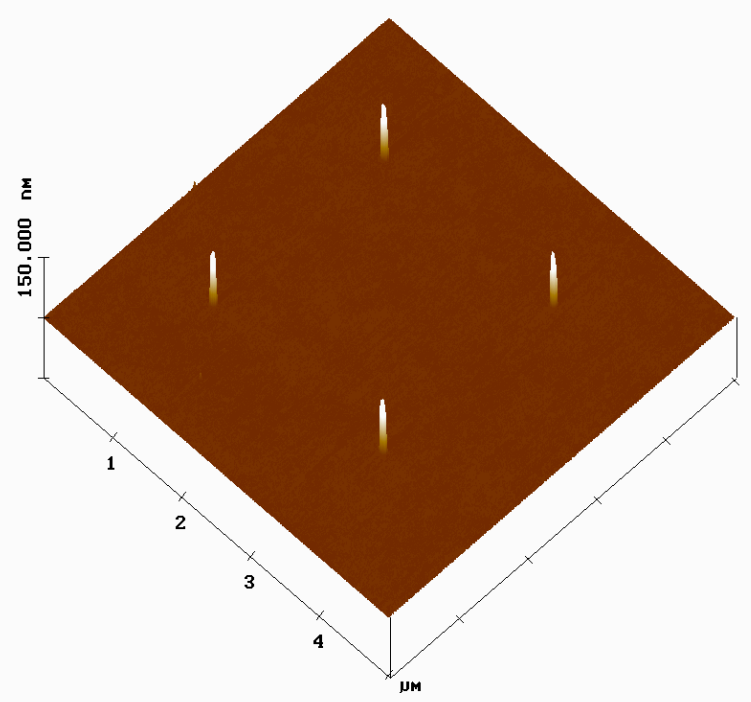

Figure 1.

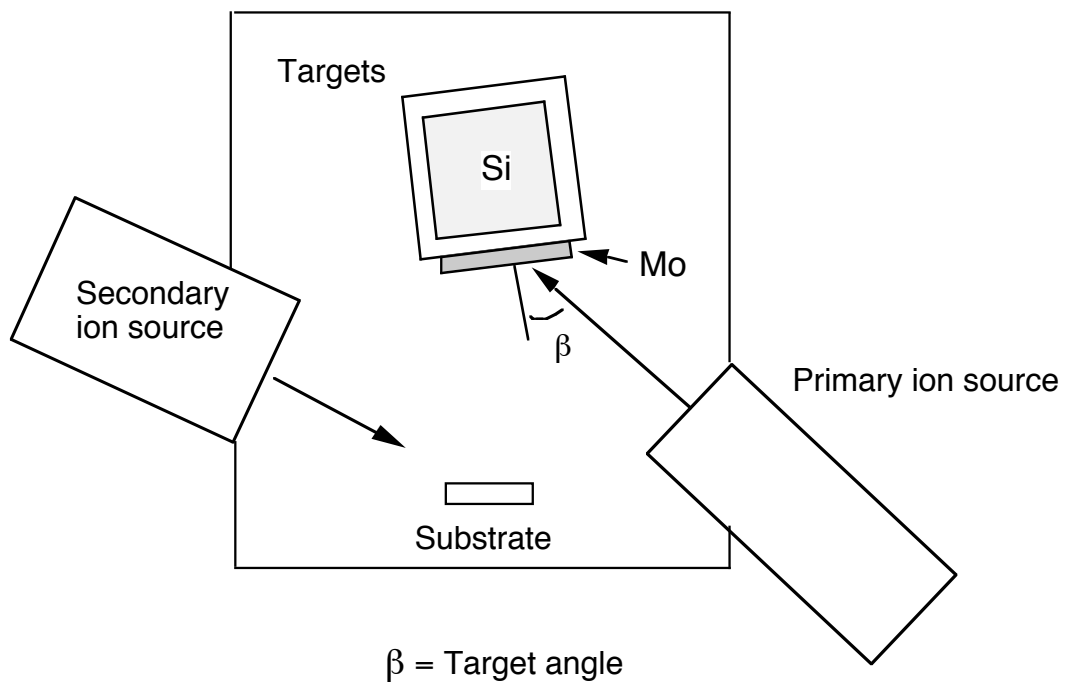

Figure 2. 
Deposit at near-normal incidence

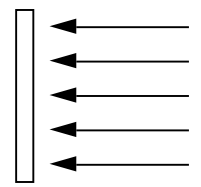

Rotate and etch at near-normal incidence

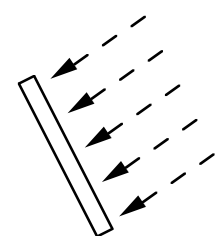

Rotate back and deposit

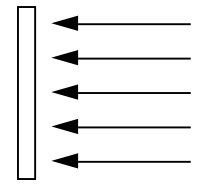

Figure 3.

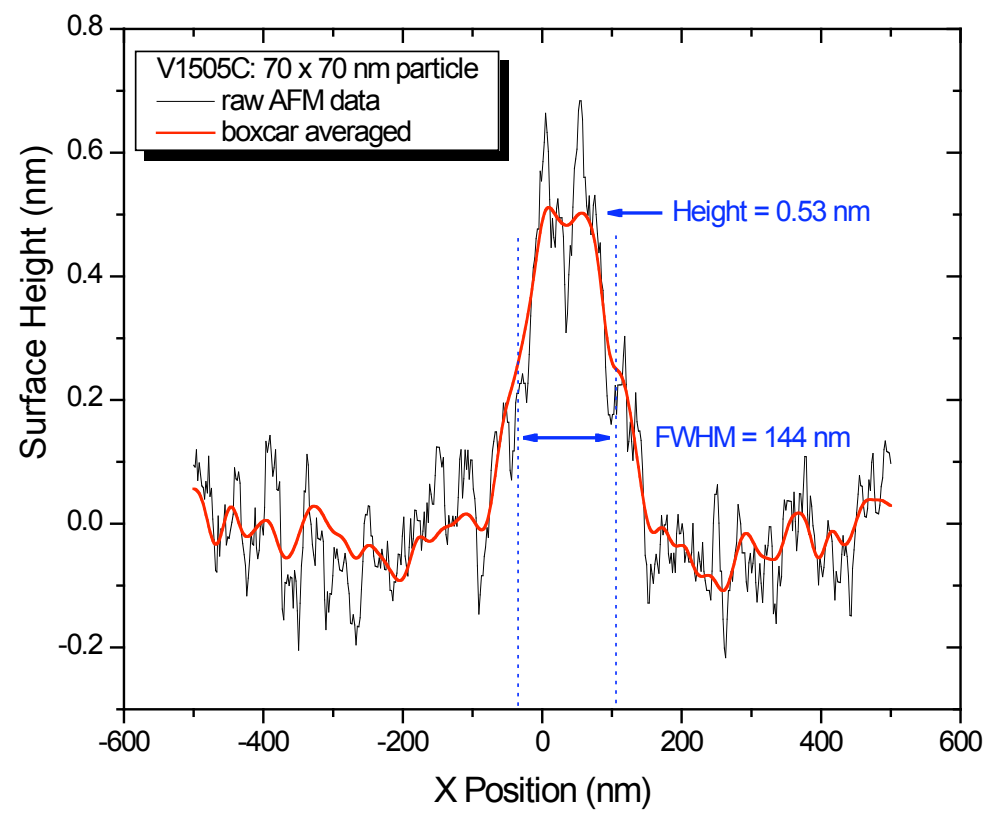

Figure 4. 


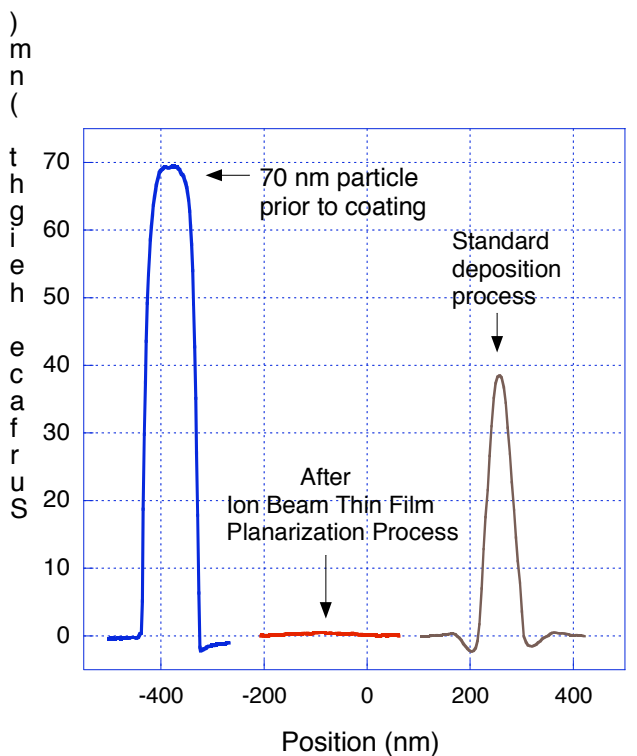

(a)

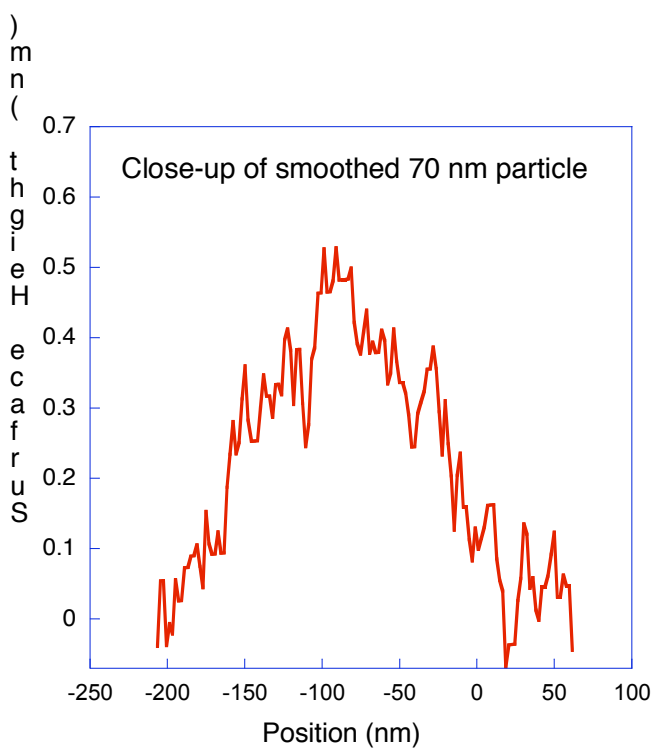

(b)

Figure 5.

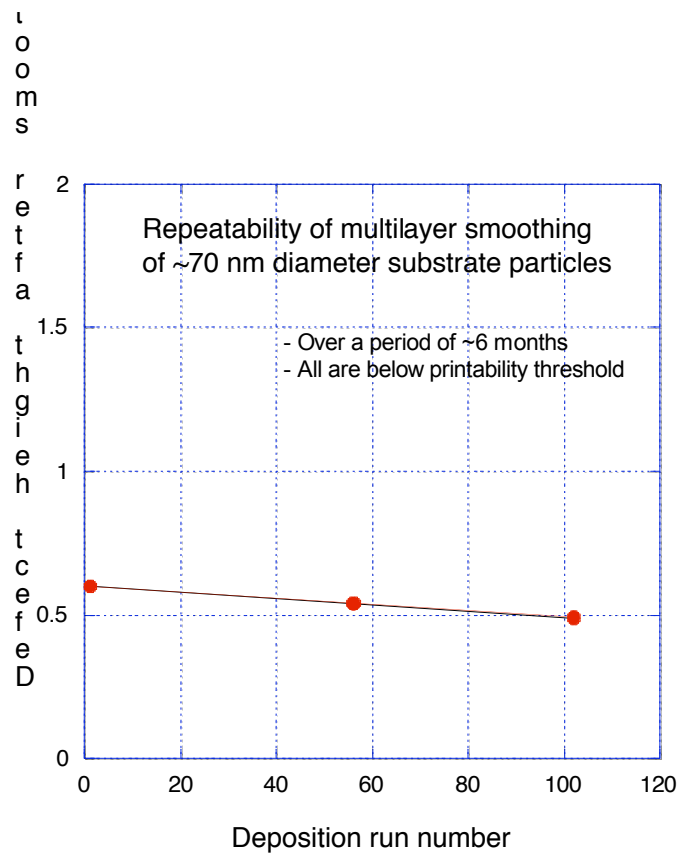

Figure 6. 


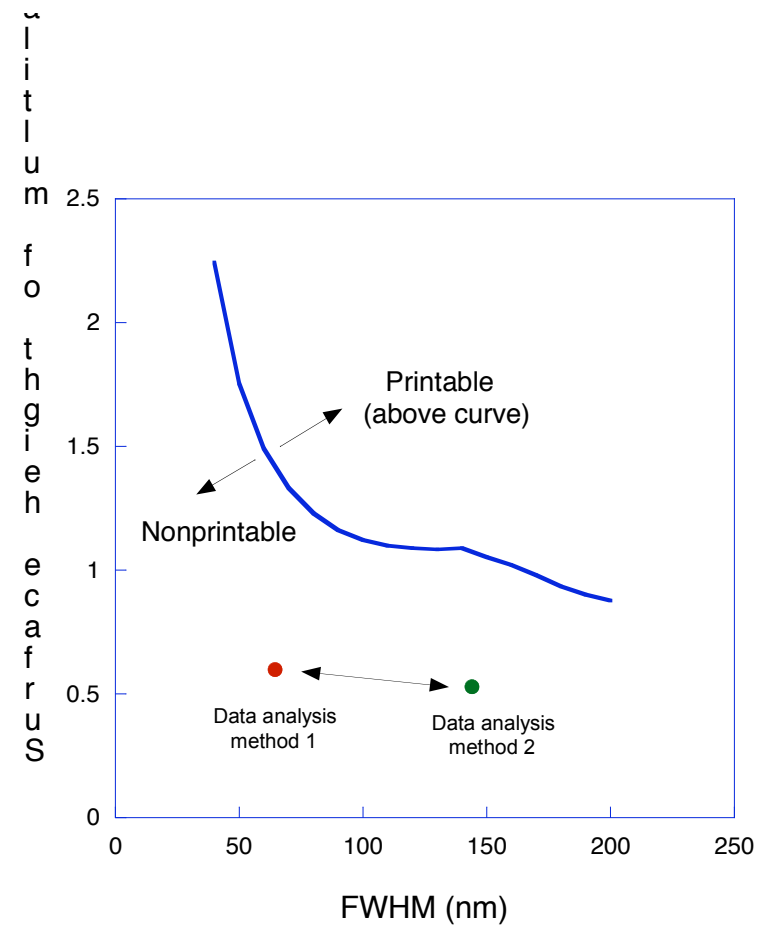

Figure 7. 


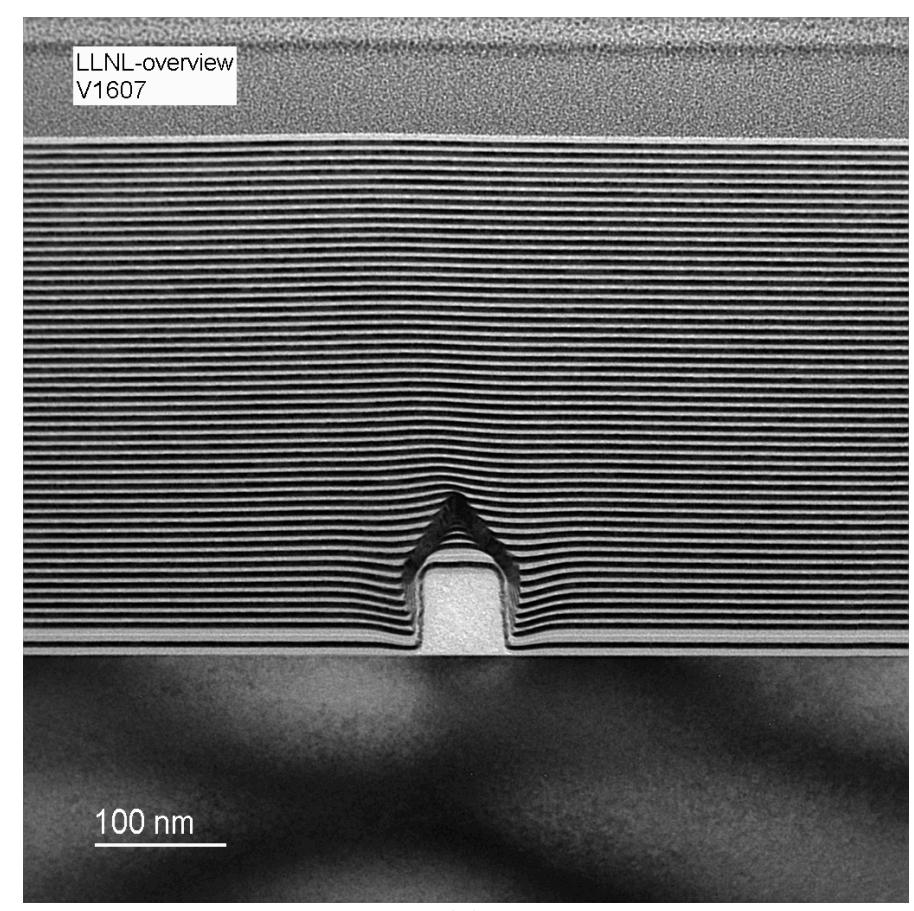

(a)

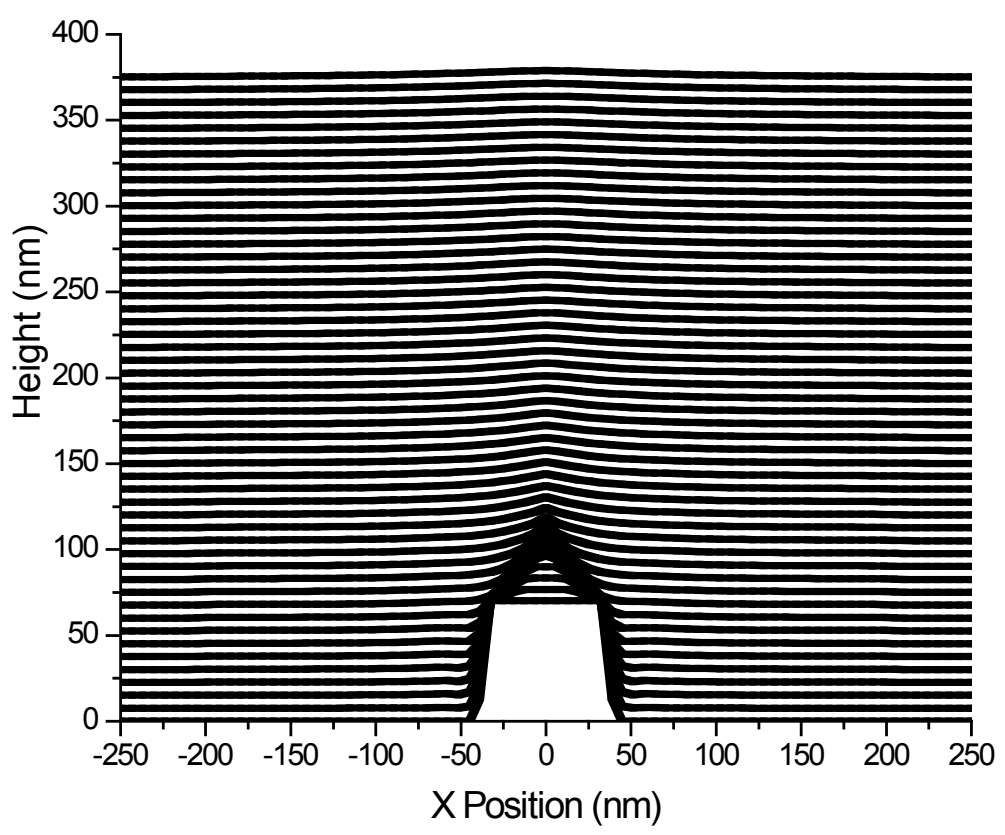

(b)

Figure 8 . 


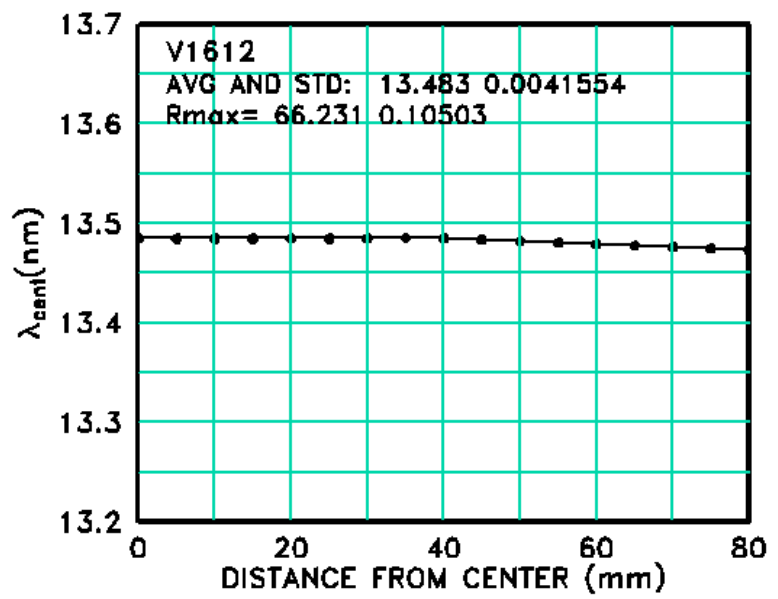

(a)

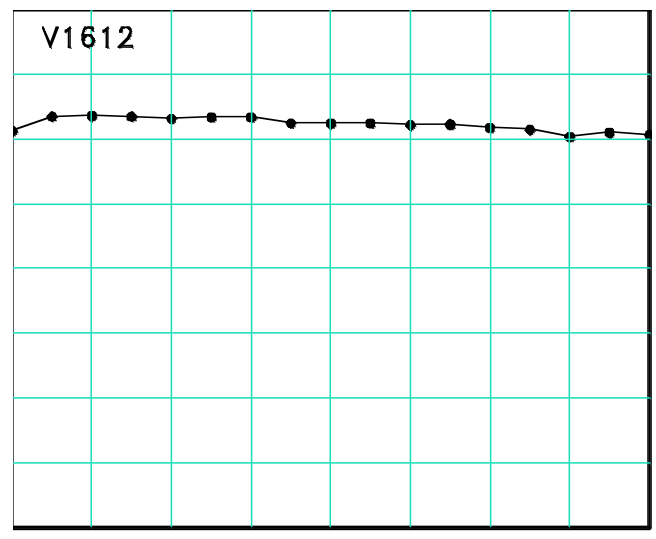

(b)

Figure 9. 


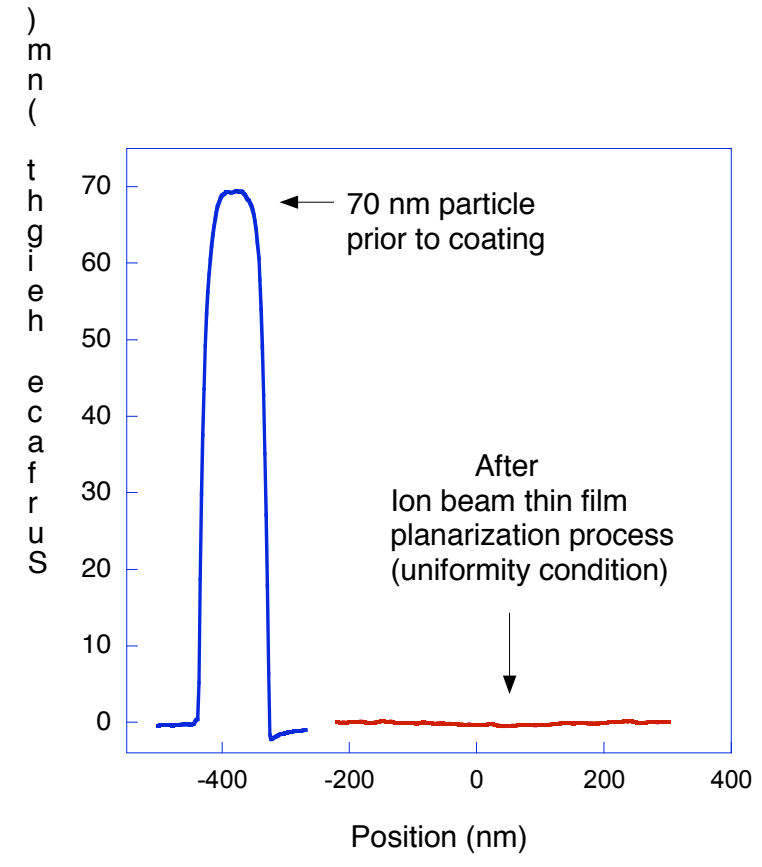

(a)

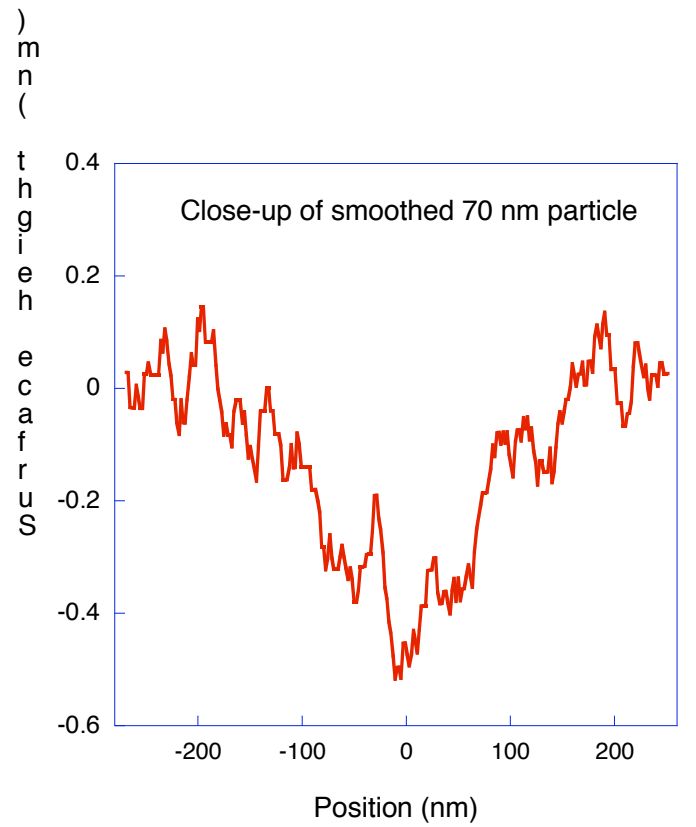

(b)

Figure 10.

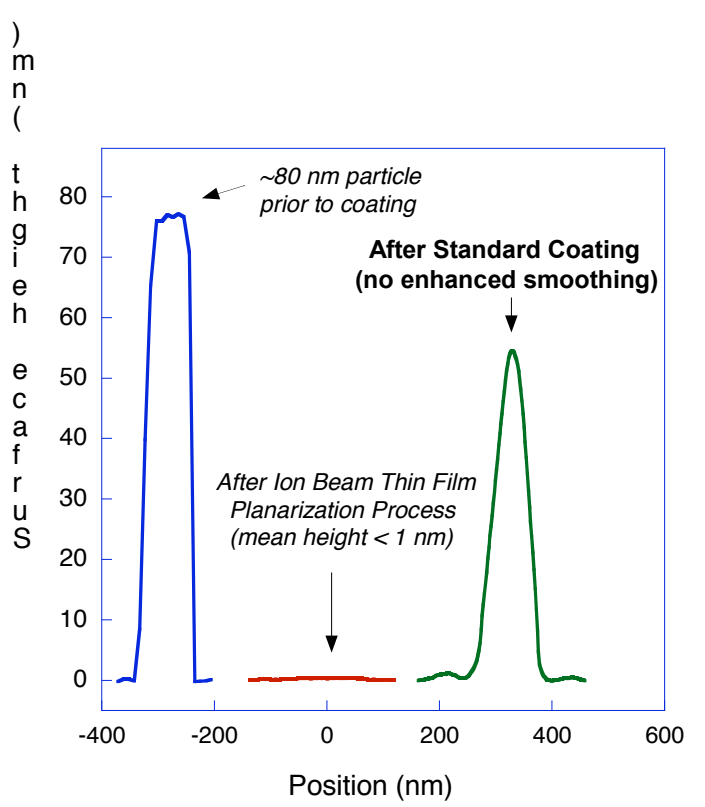

(a)

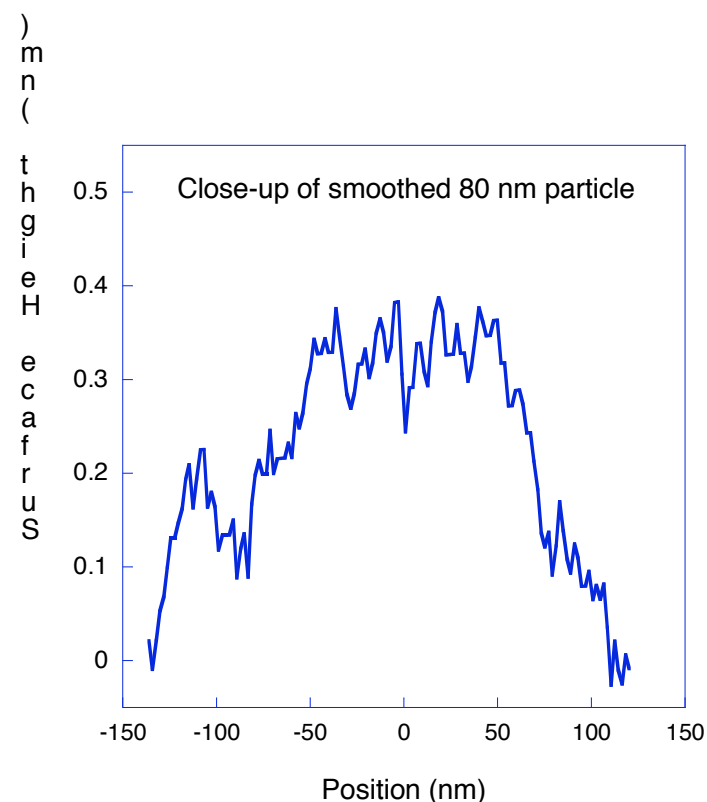

(b)

Figure 11. 